\title{
NON-LINEAR AMPLIFICATION OF ELECTROMYOGRAPHIC SIGNALS WITH PARTICULAR APPLICATION TO HUMAN CHEWING
}

\author{
C. S. Stohler, Y. Yamada and M. M. Ash JR \\ Stomatognathic Physiology Labozatory, Department of Occlusion, School of Dentistry, University of \\ Michigan, Ann Arbor, MI 48109-1078, U.S.A.

\begin{abstract}
Summary-A method is described for logarithmically-amplifying electromyographic signals so that the most commonly-occurring events within them are enhanced selectively before digital conversion. There is a need to resolve low and intermediate levels of activity during chewing, while preserving the occasional high-level responses. Besides achieving a higher resolution in subsequent digital sampling, this signalprocessing technique increased the representation of low and intermediate activities in polygraphic displays. It further ensured that signals were presented to the $A / D$ converter within the code width of the instrument.
\end{abstract}

\section{INTRODUCTION}

Automated processing of bioelectric signals with a wide dynamic range presents a common problem in conventional, biomedical instrumentation. During chewing, electromyographic (EMG) signals from the jaw-elevator muscles vary from a few microvolts to millivolts, and gain corrections are required to adjust the analogue signal to the reference range of the $A / D$ converter. An approximately Gaussian amplitude distribution of the conventional interference pattern electromyogram from surface electrodes was reported by Roesler (1974). When conventionally-acquired EMG signals are referenced to the code width of the $A / D$ converter, however, peak values in the data stream determine the gain factor; priority may be given to spikes which comprise a minor component of the EMG and small responses may be insufficiently represented.

Logarithmic amplification of EMG signals has been used in control systems for limb prostheses (Wirta, Taylor and Finley, 1978) but not for diagnostic application of a bipolar bioelectric signal.

\section{MATERIALS AND METHODS}

\section{System design}

Compression of wide-range analogue data was obtained by means of a buffer amplifier, rectifiers for the positive and negative part of the EMG signal, logarithmic amplifiers and a differential amplifier. As logarithmics may be computed only for positive arguments, separate processing of the positive and negative part of the signal was required. Figure 1 illustrates the connection scheme. The input data were scaled, by means of an isolation amplifier, to the range in which the logarithmic transfer function showed least logarithmic conformity errors. A plot of logarithmic input versus output on semilog paper showed a straight line error \pm 5 per cent max. R.T.I. (reference to input signal) in the middle three decades of the five decade range tested. The compressed EMG signal was adjusted to the code width of the $A / D$ converter to obtain maximum resolution in the ten bit successive-approximation process. Zero and full-scale calibration was accomplished using the static adjustment procedure. Zero was adjusted with the analogue

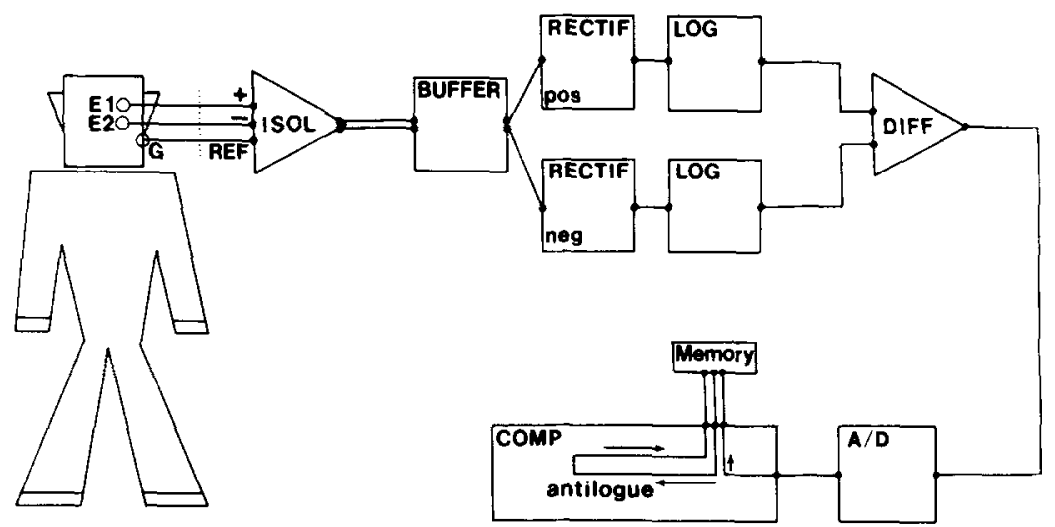

Fig. 1. Connection scheme. E1, $2=$ recording electrodes, $\mathrm{G}$ REF $=$ ground reference electrode, $\mathrm{ISOL}=$ isolation amplifier, BUFFER = buffer amplifier, RECTIF pos, neg - rectifiers for positive/negative part of signal, $L O G=$ logarithmic amplifier, $D I F F=$ differential amplifier, $A / D=$ analog-to-digital converter. COMP $=$ computer 


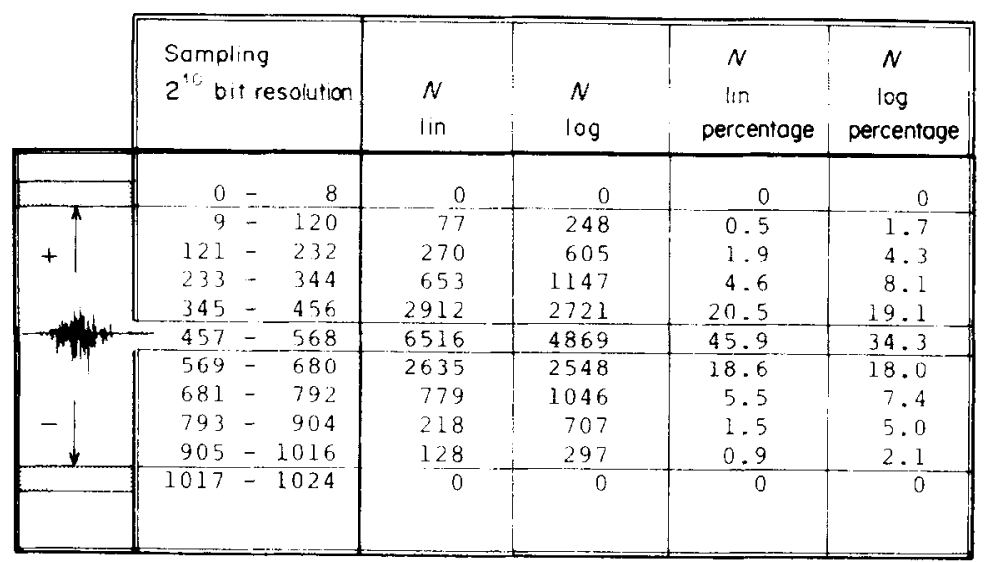

Fig. 2. Distribution of sample data-points of chewing-EMG of the right anterior temporal muscle with linear (lin) and logarithmic (log) signal processing. The chewing-EMG sample was $9.9 \mathrm{~s}$ long; the chewing frequency was $0.9 \mathrm{~Hz}$. $N$ refers to number, "o to percentage of data points. Note the better distribution of the signal over the full range in logarithmic mode.

input near the most negative end, and gain with the analogue input near the most positive end of the analogue range. The resultant digital code was directly inserted into memory. Computation of the antilogue of the voltage value was software controlled and enabled the expansion of previously- compressed data if conventional (linear) data were required.

\section{Procedure}

Bipolar surface signals of the right anterior temporal muscle were recorded. Tracking of the mandibular
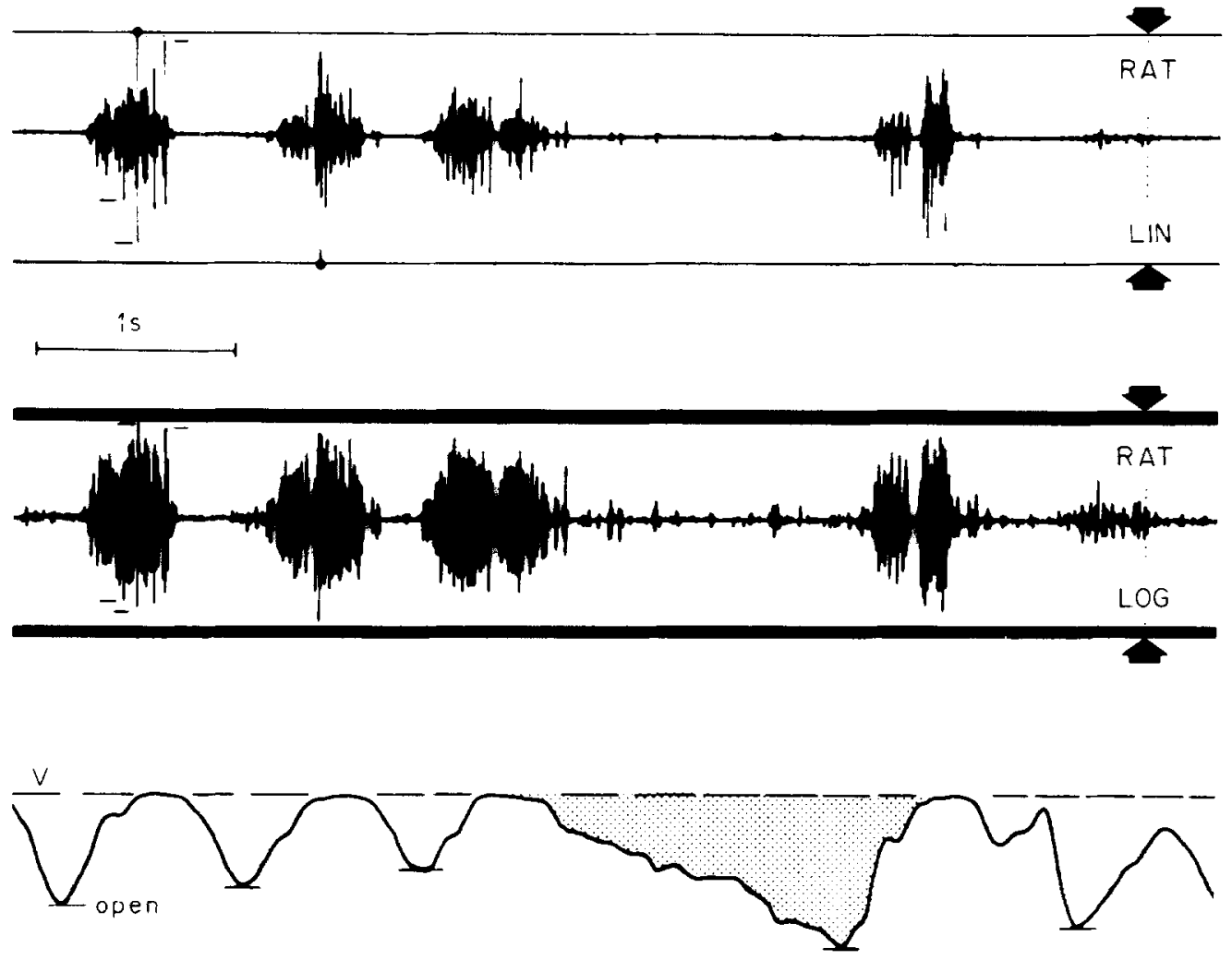

Fig. 3. Polygraphic display of a chewing sequence showing the vertical jaw-movement component and EMG of the right anterior temporal muscle in conventional (RAT-LIN) and logarithmic (RAT-LOG) mode. Both EMG data channels were adjusted to display the same voltage range. Small activities of this elevator muscle are lost in the polygraphic display in linear mode, especially during the phase, where the ongoing jaw movement was opposed to the action of this muscle. Note the representation of the interstroke activities between the major activity bursts of this elevator muscle. Time calibration: $1 \mathrm{~s}$. $\mathrm{V}=$ vertical jaw movement component. 
incisal point displacement was performed using the Mandibular Kinesiograph (Mod. MKG-5 Research, Myotronics Inc., Seattle, WA 98101). Jaw movement data were used as a reference for the nature of the signals under investigation.

\section{Signal processing}

EMG signals were either processed in the conventional (Amplifier Mod. 7P511, Grass Inc., Quincy, MA 02169) or logarithmic mode. Polygraphic plots against time of conventional (linear) and logarithmic EMG, together with the incisal point movement, were generated to compare the two techniques. The analogue continuum was partitioned into a $2^{10}$ range ( 10 bit) at a sampling rate of approx. $1.5 \mathrm{kHz}$ per data channel. Low-pass filtering $(-3 \mathrm{~dB}$ down at $560 \mathrm{~Hz}$ ) was applied according with the Nyquist criteria for signal sampling, and the frequencies expected in surface-electrode recordings (Palla and Ash, 1981).

\section{RESULTS AND DISCUSSION}

\section{Conventional data acquisition}

Appropriate A/D conversion of conventionallyacquired electromyographic data required a careful determination of the voltage range in which the observation occurred. This procedure, carried out with an analogue storage oscilloscope, was elaborate and time consuming. The presence of a few extreme voltage peaks in the EMG demanded the adjustment of the A/D converter input to these values to avoid the possibility of exceeding the input limits of the A/D converter.

\section{Logarithmic data acquisition}

EMG signals obtained during chewing and swallowing could be referenced to three decades of logarithmic amplification. Thus, the problem of signal saturation at the $A / D$ converter input could be eliminated.

\section{Comparison of the techniques}

The digital representation of the analogue continuum is shown in Fig. 2 which shows the number of data points distributed over the full-code width with linear and logarithmic amplification adjusted to the maximum code width $(+1 /-1 V)$ of the A/D converter. Results are based upon analysis of 9.9 continuous seconds of chewing data. Small and medium activities formed the dominant components of the chewing electromyogram. A polygraphic display of two chewing cycles is given in Fig. 3. The EMG record is shown in both the conventional (linear) and logarithmic mode to compare the two processing techniques. Both data channels were adjusted to display equal peak amplitude values at the polygraph. A difference in the amplitudes of the EMG bursts due to the greater amplification of small and medium activities in logarithmic mode can be seen. Another advantage of logarithmic amplification was observed in the inter-stroke sequence displayed in this record. Whereas logarithmic amplification revealed low-level muscle activity during inter-stroke movements and offered a good representation of the power stroke at the same time, the conventional (linear) technique, with the gain set to appropriately display the large activities, hardly revealed the activity between chewing strokes. Small activities, may be corrective in nature, were easily overlooked in the conventional format during movements which were opposed to the action of a muscle because the amplification factor had to be adjusted during a phase where this muscle produced its maximum output.

Besides offering these advantages, all previously used data presentations based upon linearly-acquired data are readily accessible. Antilogue conversion permits to restore the conventional (linear) electromyogram from the logarithmic format for considerations or applications requiring linear data.

This approach is likely to prove useful for research in the field of mastication.

\section{REFERENCES}

Palla S. and Ash M. M. Jr (1981) Effect of bite force on the power spectrum of the surface electromyogram of human jaw muscles. Archs oral Biol. 26, 287-295.

Roesler H. (1974) Statistical analysis and evaluation of myoelectric signals for proportional control. In: The Control of Upper-Extremity Prostheses and Orthoses (Edited by Herberts P.). Thomas, Springfield, IL.

Wirta R. Taylor D. and Finley R. (1978) Pattern recog nition arm prosthesis: A historical perspective - A final report. Bull. prosthe1. Res. 10-30, 8-34. 